Vittalle - Revista de Ciências da Saúde v. 32, n. 2 (2020) 36-45

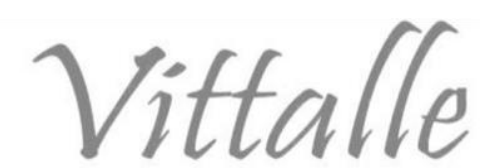

\title{
Caracterização das cesarianas em um Hospital Público do Distrito Federal
}

\author{
Geralda Carolina Alves ${ }^{\mathrm{a}}$, Ivone Kamada ${ }^{\mathrm{b}}$, Hygor Alessandro Firme Elias ${ }^{\mathrm{c}}$, Natália \\ Jardim de Carvalho Schettini ${ }^{\mathrm{d}}$, Thereza Cristina de Souza Mareco ${ }^{\mathrm{e}^{*}}$ \\ aPrograma de Mestrado Profissional em Saúde Coletiva da Universidade de Brasília, Brasília, DF, Brasil \\ bPrograma de Pós-graduação em Enfermagem, Universidade de Brasília, DF, Brasil \\ ${ }^{\mathrm{c}}$ Casa de Parto de São Sebastião (SES-DF), Brasília, DF, Brasil; Tribunal de Justiça do Distrito Federal e Territórios - \\ TJDFT, Brasília, DF, Brasil \\ ${ }^{\mathrm{d}}$ Hospital Materno Infantil de Brasília, Brasília, DF, Brasil \\ ${ }^{\text {e}}$ Fundação de Ensino e Pesquisa em Ciências da Saúde (SES-DF), Brasília, DF, Brasil. \\ Histórico do Artigo \\ Recebido em: \\ 08/01/2020 \\ Aceito em: \\ $25 / 03 / 2020$ \\ Palavras-chave: \\ Cesárea; trabalho de \\ parto; enfermagem

\begin{abstract}
RESUMO
Objetivou-se descrever as principais indicações das cesarianas realizadas no Hospital da Região Leste (HRL) do Distrito Federal. Trata-se de um estudo descritivo observacional retrospectivo realizado em um hospital público do DF, no período de janeiro a dezembro de 2014, identificando as indicações de cesariana, contrapondo-as às evidências científicas. Projeto de pesquisa aprovado pelo Comitê de Ética em Pesquisa, CAAE 47375615.0.0000.5553. A taxa de cesarianas foi de $43,8 \%$. As variáveis obstétricas que apresentaram maior associação com a cesárea foram: $30 \%$ cesárea prévia, $15 \%$ sofrimento fetal agudo e $8 \%$ desproporção cefalopélvica. A incidência de cesáreas foi maior que o índice recomendado pela OMS e Ministério da Saúde. Questiona-se a falta de protocolos e fluxogramas para definição de diagnósticos e condutas, fazendo uso da subjetividade e decisão individual do profissional. Espera-se que os resultados apresentados subsidiem uma revisão nas condutas e critérios de indicação das cesáreas, buscando a melhoria da assistência prestada às parturientes.
\end{abstract} obstétrica

Keywords:

Cesarean section; labor; obstetric nursing
Characterization of cesarean sections in a public hospital in the Distrito Federal

\section{ABSTRACT}

The objective of the study was to describe the main indications of cesarean sections performed at the Eastern Region Hospital (HRL) of the Federal District. It was a retrospective observational descriptive study carried out in a public hospital in DF, from January to December 2014, identifying as indications for cesarean section, contrasting them with scientific research. Research project approved by the Research Ethics Committee, CAAE 47375615.0.0000.5553. The cesarean section rate was $43.8 \%$. The obstetric variables that presented the greatest association with cesarean section were: $30 \%$ previous cesarean section, $15 \%$ acute fetal distress and $8 \%$ cephalopelvic disproportion. The incidence of cesarean sections was higher than the index recommended by the WHO and the Ministry of Health. The lack of protocols and flowcharts to define diagnoses and behaviors is questioned, due to the subjectivity of the individual's professional decision. It is hoped that the presented results will subsidize a revision in the conducts and criteria of cesarean indication, seeking the improvement of the care provided to the parturient.

\section{Introdução}

A cesariana é um procedimento cirúrgico onde é feita uma incisão na parede abdominal e uterina para a extração fetal. O termo cesárea deriva do verbo em latim caedere, que significa corte, e possui origens discutíveis. Os primeiros registros de cesariana envolvem lendas e relatos da mitologia greco-romana, como o nascimento de Esculápio, Deus da medicina que teria sido retirado do ventre de sua mãe, por Apolo, seu pai. Também há transcrições de que o líder da República Romana, Júlio César, teria 
sido extraído do útero de sua mãe após sua morte, dando origem ao nome cesárea (1).

Atualmente, o Brasil é o país que mais realiza cesarianas no mundo, principalmente na rede suplementar, comparando com o parto normal, as taxas de cesárea chegam a $84 \%$ no sistema privado e a $40 \%$ no Sistema Único de Saúde (SUS). A OMS recomenda a taxa ideal de cesárea entre $10 \%$ e $15 \%$ e que deve realizada somente em situações nas quais o parto normal não seja recomendado (2-5).

Estudos mostram que os benefícios conferidos ao feto pela cesariana são pequenos, por isso, a alta incidência desta cirurgia tem sido motivo de muitos questionamentos $(3,6)$. A cesariana eletiva pode causar prematuridade iatrogênica, prejudicar a amamentação e prolongar a internação, elevando os custos para o sistema e para as famílias. Também implica em riscos cirúrgicos, anestésicos e consequências nas gestações subsequentes (3,7-9).

A mortalidade materna na cesariana é de quatro a cinco vezes maior que no parto vaginal e está associada ao aumento da morbimortalidade perinatal (10,7). Visto isso, a Agência Nacional de Saúde Suplementar (ANS) divulgou em janeiro de 2015 na Resolução Normativa $n^{\circ} 368$ novas regras para a redução do número de cesarianas eletivas e estímulo ao parto normal, entre elas a restrição ao agendamento de cesáreas eletivas, onde mesmo que a mulher opte por um parto cirúrgico, este deve ser feito após ter iniciado o trabalho de parto espontâneo $(9,11,12)$.

A tensão e o medo da dor, aliados à falta de orientação e apoio profissional, faz com que as mulheres optem pelo parto cirúrgico e o vejam como alívio do sofrimento de horas de trabalho de parto, além de transmitir uma errônea sensação de segurança para todos os envolvidos, aumentando a sua banalização $(9,13-15)$.

O Ministério da Saúde (MS) tem demonstrado preocupação com o crescimento de partos cirúrgicos e tem buscado estratégias para a redução das taxas de cesariana no Brasil. Assim, em 2017 foi criado o projeto Apice On (Aprimoramento e Inovação no Cuidado e Ensino em Obstetrícia e Neonatologia), uma iniciativa em parceria com outras instituições, em busca de ações para promover a qualificação de serviços através da disseminação de estratégias para mudanças nos modelos tradicionais de formação, atenção e gestão, com foco em hospitais com atividades de Ensino, tornando-os referência nas melhores práticas de atenção/ cuidado ao parto e nascimento (16).

A inserção da equipe multiprofissional no parto, formação de enfermeiras obstétricas, campanhas publicitárias de incentivo ao parto normal e implantação de Centros de Parto Normal (CPN) concomitante ao movimento a favor da Humanização do Parto e Nascimento são alguns dos investimentos para redução desses índices $(7,13,14)$.

Considerando o contexto, este estudo teve como objetivo caracterizar as principais indicações de cesarianas no Hospital da Região Leste (HRL) do Distrito Federal, levando em consideração as evidências científicas a respeito.

\section{Métodos}

Trata-se de um estudo documental, descritivo observacional, retrospectivo e transversal com coleta de dados diretamente de livros de registro e indicadores de parto, prontuários físicos e eletrônicos das mulheres que realizaram cesariana no período de janeiro a dezembro de 2014, no Centro Obstétrico do HRL.

Os dados foram analisados de forma univariada, por meio de estatística descritiva através do cálculo de frequências absolutas e relativas em um banco de dados gerado no programa Microsoft Office Excel 2007.

A planilha elaborada contava com os seguintes campos: data do parto, iniciais do nome da mãe, número de registro em prontuário, dados quanto à paridade (número de 
filhos, tipos de parto e ano e mês do último parto), além da indicação médica registrada para a prescrição da cesariana.

O único critério de inclusão adotado foi de parturientes que foram submetidas a cesariana no período em estudo. Esta pesquisa foi aprovada pelo Comitê de Ética em Pesquisa da Fundação de Ensino e Pesquisa em Ciências da Saúde (FEPECS), com CAAE $n^{\circ}$ 47375615.0.0000.5553, e dispensada do Termo de Consentimento Livre e Esclarecido (TCLE) conforme estabelecido na resolução do Conselho Nacional de Saúde 466/2012 e suas complementares.

\section{Resultados}

No ano de 2014 o Centro Obstétrico do HRL atendeu 2948 nascimentos, sendo 1291 $(43,8 \%)$ por cesarianas. Mesmo que este estudo tenha sido realizado há alguns anos, ainda retrata a realidade enfrentada no hospital público representado, visto que mesmo após as iniciativas do MS e a implementação do projeto Apice On, essa taxa de cesarianas foi superada, apesar de não ter ultrapassado $50 \%$ nos últimos anos.

$\mathrm{Na}$ análise dos prontuários foram encontrados 33 tipos de indicações clínicas e/ou obstétricas para justificar a realização da cesárea, entre elas as mais prevalentes estão relacionadas na Tabela 1.

Tabela 1 - Principais indicações de cesariana no HRL, Brasília-DF, 2014 (n=1291)

\begin{tabular}{lcc}
\hline Indicação & N & \% \\
\hline Cesárea prévia & 388 & 30 \\
Sofrimento fetal agudo & 192 & 15 \\
Desproporção cefalopélvica & 103 & 8 \\
Parada de progressão & 81 & 6 \\
Síndrome hipertensiva & 80 & 6 \\
Apresentação pélvica & 61 & 5 \\
Macrossomia fetal & 43 & 4 \\
Oligodramnia & 43 & 4 \\
Falha de indução & 41 & 3 \\
Sem registro da indicação & 54 & 4 \\
Outras & 205 & 15 \\
Total & $\mathbf{1 2 9 1}$ & $\mathbf{1 0 0}$ \\
\hline
\end{tabular}

Quanto ao intervalo entre a gestação atual e cesárea anterior, 46,6\% das mulheres tinham mais de dois anos de intervalo entre as cirurgias; $22,2 \%$ com intervalo menor e em $31,2 \%$ dos casos, este dado não foi encontrado.

$\mathrm{O}$ sofrimento fetal agudo (SFA), segunda indicação mais frequente, engloba na cardiotocografia (CTG) as seguintes subcategorias: feto hipoativo e hiporreativo, CTG padrão comprimido, taquicardia e bradicardia fetal persistentes e desacelerações tardias e variáveis.

No estudo, em $72 \%$ dos recém-nascidos (RNs) que nasceram por cesariana em função de SFA, o índice de Apgar foi maior ou igual a 8 no $1^{\circ}$ minuto de vida, $14,5 \%$ tiveram Apgar entre 7 e 5, 7,8\% menor que 4 e em 5,7\% a informação não foi encontrada. Ainda em relação às indicações por SFA, em 120 casos o líquido amniótico (LA) estava claro e nos outros 72 havia líquido amniótico meconial (LAM), ainda assim, a maioria destes RNs (66,6\%), nasceram com Apgar maior ou igual a 8, como mostra a Tabela 2. 
Tabela 2 - Apgar no $1^{\circ}$ minuto de vida de RNs que estavam sob LAM no Hospital da Região Leste. Brasília (DF), Brasil, 2014 (n=72).

\begin{tabular}{ccc}
\hline Apgar & $\mathbf{N}$ & \% \\
\hline$\geq 8$ & 48 & 66,6 \\
$7-5$ & 15 & 21 \\
$\leq 4$ & 6 & 8,3 \\
Sem informação & 3 & 4,1 \\
Total & $\mathbf{7 2}$ & $\mathbf{1 0 0}$ \\
\hline
\end{tabular}

As cesarianas prescritas por síndromes hipertensivas incluem a hipertensão arterial gestacional transitória e crônica, pré-eclâmpsia leve e grave, eclampsia e síndrome HELLP, as quais correspondem ao total de 80 casos $(6,2 \%)$.

Ainda dentre as indicações, 43 (3,3\%) foram por macrossomia fetal, onde $60,5 \%$ dos RNs tinham peso normal e apenas 17 (39,5\%) eram realmente considerados macrossômicos, com peso ao nascimento maior ou igual a $4000 \mathrm{~g}$.

Além disso, foi observado que o dia e o horário de nascimento influenciam diretamente sobre a via de nascimento. Houve um menor número de cesáreas durante a madrugada (entre 00h e 06h59) e aos finais de semana, conforme Tabela 3.

Tabela 3 - Período e dias da semana de realização das cirurgias no Hospital da Região Leste. Brasília (DF), Brasil, 2014 ( $\mathrm{n}=1291)$.

\begin{tabular}{lcc}
\hline Período & Número de cesáreas (n) & \% \\
\hline Manhã & 385 & 29,8 \\
Tarde & 401 & 31,2 \\
Até 00h & 384 & 29,7 \\
00h01 a 06h59 & 121 & 9,3 \\
\hline
\end{tabular}

\begin{tabular}{lc}
\hline Dias da semana & Número de cesáreas (n) \\
\hline Segunda-feira & 206 \\
Terça-feira & 191 \\
Quarta-feira & 207 \\
Quinta-feira & 194 \\
Sexta-feira & 197 \\
Sábado & 159 \\
Domingo & 137 \\
Total & $\mathbf{1 2 9 1}$ \\
\hline
\end{tabular}

\section{Discussão}

Este estudo revela o alto índice de cesarianas registrado na instituição pesquisada, que supera as definições preconizadas pela OMS e MS. Também se dispõe a analisar e discutir as principais indicações, além de correlacioná-las com as evidências científicas a respeito desse procedimento.

\section{Cesárea prévia}

A presença de cesariana anterior foi a principal justificativa para repetição deste procedimento e está em concordância com estudos semelhantes $(2,10,17,15)$. Ocorre principalmente devido ao receio que os médicos possuem da ocorrência de ruptura uterina, entretanto, evidências científicas consideram nível A (altamente recomendável (5)) a tentativa de parto vaginal após cesárea, uma vez que a chance de sucesso é de 
$70 \%$ e a de ruptura é de $0,16 \%$ a $2,1 \%(6,18)$.

Há vários benefícios maternos no parto vaginal após cesárea, entre eles: recuperação mais rápida, menor intensidade da dor no pós-parto, redução no tempo de internação, e menor risco futuro de outra cesárea $(3,6,18)$.

A maioria das mulheres submetidas à realização de cesariana por esta indicação foi de forma eletiva, tendo como único critério a gestação a termo. Nesses casos, a literatura recomenda que se espere o desencadeamento do trabalho de parto espontâneo ou até considerar a possibilidade da indução do parto com métodos que apresentam menores riscos, preferencialmente os mecânicos e a ocitocina (18).

Ademais, estudos evidenciam que o tempo necessário para o organismo materno se restabelecer após um parto (vaginal ou cesáreo) é em média 18 meses (19). Na unidade em estudo. Em alguns casos, o intervalo interpartal chegou a ser superior a 20 anos, evidenciando um círculo vicioso, onde a ocorrência de uma cesárea condiciona a repetição desta prática, independente do intervalo passado, além de não permitir a prova de trabalho de parto a essas mulheres e nem considerar o desejo das mesmas $(14,18,20)$.

\section{Sofrimento fetal agudo - SFA}

Os batimentos cardíacos fetais (BCF), aspecto do LA e movimentação fetal são as principais condições que refletem o bem-estar fetal. Situações como LAM e alterações na CTG podem caracterizar o SFA, condição a qual se mostra com grande influência para o nascimento por via alta, sendo o segundo principal motivo de realização de cesariana neste estudo.

Sinais de SFA, como hipoxemia e redução persistente do BCF, quando não melhoram com a mudança de decúbito e controle da respiração materna, podem indicar que o feto não apresenta reserva metabólica para suportar o trabalho de parto, por isso o nascimento deve ser pela via mais rápida. Ainda assim requer um diagnóstico cuidadoso, com realização de CTG sem que a mãe esteja em jejum e amostra do pH fetal quando possível $(2,21)$.

A presença isolada de LAM fluido não indica SFA, geralmente está associada à maturidade fetal (21). Sua presença deve ser considerada um sinal de alerta, exigindo monitoração criteriosa do BCF. Já na presença de mecônio espesso, deve-se usar a CTG contínua, avaliar a dilatação cervical, altura da apresentação e variedade de posição, havendo dilatação completa, indica-se o uso de fórceps ou o vácuo-extrator $(2,21)$. Em último caso, se for realmente identificado SFA e com o colo desfavorável, a conduta a ser tomada deve ser a cesariana (21).

O escore de Apgar é uma escala de avaliação da vitalidade fetal que vai de 0 a 10 , onde valores inferiores a 7 podem confirmar o diagnóstico de SFA e avaliar se a indicação de cesariana foi adequada (22).

Neste estudo, entre as indicações por SFA, 72\% dos RNs obtiveram Apgar maior ou igual a 8 no primeiro minuto de vida, notando-se o equívoco no diagnóstico.

\section{Desproporção céfalopélvica (DCP)}

A desproporção entre o feto e a pelve materna, ocorre em mulheres que possuem alguma deformidade ou desalinho nos ossos pélvicos, devido a deficiência física, variações anatômicas da pelve ou em casos em que o feto é grande, por hidrocefalia ou macrossomia (6,23). A DCP representa uma das mais frequentes indicações de cesariana e talvez a mais discutível, entretanto quando bem diagnosticada é uma das únicas indicações absolutas da cesariana (2). 
Neste estudo, a DCP foi responsável por $8 \%$ das indicações de cesáreas. Este diagnóstico deve ser baseado no partograma, por ser a representação gráfica da evolução do trabalho de parto (13). Contudo, este importante instrumento praticamente não é usado na instituição, sua utilização restringe-se aos fins de aprendizagem, para residentes de enfermagem e alunos de estágio.

\section{Parada de progressão}

Em geral, a falha na progressão do trabalho de parto se deve a ineficiência ou falta das contrações uterinas, podendo ainda estar relacionada a uma DCP absoluta ou relativa. A primeira intervenção indicada nesses casos é o estímulo às contrações, através da adoção de posições verticalizadas, exercícios pélvicos, ruptura artificial das membranas e/ou a administração de ocitocina, sempre com assistência obstétrica contínua $(13,18)$.

A parada de progressão representou $6 \%$ das indicações, com 81 casos. Cabe ressaltar a subjetividade na avaliação e diagnóstico, já que a maioria da equipe não utiliza o partograma, além disso, a avaliação das contrações e o toque vaginal são realizados por diferentes profissionais durante o trabalho de parto, o que pode invalidar as indicações de cesárea por parada de progressão.

\section{Apresentação pélvica}

Nesses casos, a decisão sobre a via de parto deve levar em consideração o desejo da mãe e a experiência do obstetra. Infelizmente, muitos ainda não possuem segurança, experiência e habilidade para condução do parto pélvico e optam por indicar a cesariana $(2,6)$.

É recomendada versão cefálica externa (VCE) com 36 semanas de gestação, na ausência de trabalho de parto ou complicações maternas e/ou fetais. A cesariana deve ser indicada após a falha da VCE. Porém apesar de sua comprovada efetividade e segurança, ainda é uma técnica impopular no Brasil, sendo realizada por poucos profissionais (21).

Isto significa que a quase totalidade das gestantes com feto em apresentação pélvica no termo será indicado a cesariana, mas ainda assim, é recomendado aguardar o desencadeamento do trabalho de parto ou até a $39^{a}$ semana de gestação $(6,21)$.

No presente estudo, a maioria dos casos de apresentação pélvica teve resolução por via alta e antes do desencadeamento do trabalho de parto. Os RNs pélvicos que nasceram por parto vaginal foram "acidentais", em função da parturiente ter dado entrada no hospital já em período expulsivo, não havendo tempo hábil para a realização da cirurgia. Portanto, é notório o medo e a falta de treinamento das equipes para a assistência ao parto pélvico.

\section{Síndromes hipertensivas}

Segundo a OMS, a hipertensão arterial na gestação constitui a primeira causa de mortalidade materna no Brasil e caracteriza-se como uma intercorrência obstétrica que muito contribui para o aumento das taxas de cesariana $(6,23)$.

As síndromes hipertensivas representaram a quinta maior justificativa para realização de cesariana neste estudo e cabe ressaltar que em muitos casos foram encontrados registros de picos hipertensivos isolados, não sendo, portanto, real indicação para a cirurgia de urgência.

Mesmo com o diagnóstico firmado, o parto normal é preferível à cesariana para mulheres com pré-eclâmpsia/eclâmpsia, pois evita o estresse adicional de uma cirurgia 
em uma situação que já possui alterações fisiológicas múltiplas (23).

\section{Oligoâmnio}

O LA é fundamental para a manutenção da gestação por proteger o feto contra impactos, favorecer a movimentação fetal, além de auxiliar na formação dos sistemas muscular, respiratório, digestivo e urinário. Seu volume é avaliado através de ultrassonografia, a partir da medida em centímetros do maior bolsão vertical (21).

Na presença de oligoâmnio, a decisão de interromper a gestação e a via de parto deve considerar a etiologia da redução do LA, se é um evento isolado ou tem outros fatores associados $(21,17)$. O oligoâmnio isolado não justifica a realização de cesariana, mesmo assim representou $4 \%$ das indicações do estudo. Na maioria dos prontuários constava como causa isolada, sendo desta forma uma indicação equivocada para a realização da cirurgia.

\section{Macrossomia}

A definição de recém-nascido macrossômico (RNM) é variável na literatura, porém a maioria conceitua a macrossomia quando o $\mathrm{RN}$ apresenta peso superior a $4.000 \mathrm{~g}$ ao nascimento. Há um consenso de que nestes casos a morbimortalidade neonatal está aumentada, devido aos riscos de hipoglicemia, hipóxia neonatal e distócias intraparto (24).

O diagnóstico deve ser feito com ultrassonografia concomitante ao gráfico peso em relação a idade gestacional, presente na maioria dos cartões de pré-natal. Fetos com peso acima do percentil 95 em relação à respectiva idade gestacional são considerados macrossômicos. O diabetes mellitus gestacional (DMG) e elevado ganho de peso materno durante a gestação estão associados à macrossomia. Outras possíveis causas da macrossomia são multiparidade, idade materna avançada e pós-datismo $(2,24)$.

Nesta pesquisa, foram identificadas falhas no diagnóstico de macrossomia fetal, pois a maioria dos fetos com este diagnóstico nasceu com peso inferior a 4.000g.

\section{Falha de indução}

A indução do parto é a estimulação artificial do estabelecimento de contrações uterinas efetivas antes de seu início espontâneo, desencadeando o trabalho de parto. As condições cervicais devem ser avaliadas antes de se iniciar a indução, calculando-se o índice de Bishop quando se optar por métodos farmacológicos de indução. A falha de indução é definida quando o trabalho de parto não é deflagrado, mesmo mediante a utilização de tais métodos (20).

Nota-se a necessidade da utilização de protocolos na instituição, principalmente para os casos de pós-datismo e ruptura prematura de membranas, situações estas em que é preconizado a indução química do trabalho de parto. Identificou-se também divergências no tempo de intervalo do uso do Misoprostol 25mg, recurso farmacológico importante nas induções de trabalho de parto, especialmente para o preparo da cérvice. Alguns profissionais prescrevem-no com intervalos de seis horas entre os comprimidos, já outros preferem intervalar oito ou doze horas na prescrição.

Além disso, observa-se que o cálculo do índice de Bishop não é muito usado como critério para se iniciar a indução, sendo esta prescrita de acordo com as condutas individuais de cada profissional. O diagnóstico de falha na indução segue o mesmo preceito, alguns indicam a falha após o uso de três comprimidos, outros quatro ou cinco. Em alguns casos houve o registro no prontuário da falta de Misoprostol na unidade, 
sendo a ocitocina prescrita com colo desfavorável, o que não é indicado.

A variável falha de indução foi encontrada em 41 casos, correspondendo a 3\% das indicações de cesariana.

\section{Indicações não encontradas}

A Secretaria de Saúde do Distrito Federal realiza os registros em prontuários eletrônicos, os quais demonstram falhas ficando usualmente fora do ar. Quando isso ocorre, as evoluções passam a ser registradas manualmente e por isso as informações acabam se perdendo. $\mathrm{O}$ motivo das cesáreas sem indicações se deve principalmente a este fato.

No que se refere aos registros em prontuários, observa-se que algumas informações não foram registradas ou estavam incompletas, tais como o motivo da indicação e sobre o tempo passado após o último parto, onde em 31,2\% dos casos, o dado não foi encontrado. Este fato, evidencia a falha da equipe nas anotações nos prontuários, desconsiderando a importância destas informações.

\section{Considerações Finais}

Um ponto positivo neste estudo foi que a maioria das cesarianas $(50,5 \%)$ foram realizadas em gestantes com fetos de idade gestacional entre 39 e 40 semanas e seis dias, o qual representa o período mais favorável para a adaptação à vida extrauterina.

Sobre os horários e dias de nascimento, acredita-se que a redução de cesarianas na madrugada esteja associada ao período em que a equipe de trabalho está reduzida devido ao revezamento do horário de descanso. Aos finais de semana também houve redução no número de cirurgias, é importante ressaltar que as equipes médicas possuem escala fixa, salvo férias e outros afastamentos legais, o que sugere que algumas delas realizam mais cesarianas do que outras. Desta forma, infere-se que o período da madrugada e finais de semana foram fatores favorecedores do parto normal.

Em relação ao manejo do trabalho de parto, principalmente em relação à equipe médica, nota-se que práticas consideradas prejudiciais, como jejum, restrição ao leito e dificuldades em aceitar a presença do acompanhante são comuns no setor.

É notável a necessidade da implantação de protocolos na instituição, que além de auxiliar na definição das condutas e diagnósticos, fornecem segurança e respaldo aos profissionais e podem auxiliar na redução do número de cesarianas. Ademais, sugere-se a utilização do sistema de classificação de Robson, que permite realizar comparações na mesma instituição ao longo do tempo e favorece a identificação de grupos de mulheres que devam ser alvo de estratégias para redução de cesarianas.

Mudanças no modelo de assistência à gestação e ao parto com acolhimento e orientações à gestante durante o pré-natal, respeito à fisiologia do parto, uso das boas práticas e inserção da enfermeira obstétrica no parto são medidas importantes para redução das taxas de cesarianas.

A presença da enfermeira obstétrica na assistência ao parto e nascimento busca respeitar a fisiologia do trabalho de parto e parto, evitando intervenções desnecessárias e propiciando o protagonismo da mulher, permitindo que seja uma experiência mais agradável para a parturiente e seus familiares.

Nos últimos anos, a taxa de cesarianas tem se mantido em torno de 45\%, mesmo após a implementação de projetos e programas do MS que visam o aprimoramento da assistência à mulher em seus ciclos de vida, baseada em evidências científicas.

Espera-se que este estudo possa auxiliar os profissionais de saúde em seu processo 
reflexivo e na melhoria da assistência prestada às gestantes, fornecendo subsídios para aprimoramento dos critérios de indicação dos partos cirúrgicos.

\section{Referências}

1. Parente RCM, Filho OBM, Filho JR, Bottino NG, Piragibe P, Lima DT, Gomes DO. A história do nascimento (parte 1): cesariana. Femina 2010; 38(9).481-6.

2. Amorim MMR, Souza ASR, Porto AMF. Indicações de cesariana baseadas em evidências: parte I. Femina 2010; 38(8). 415-22.

3. Brasil. Ministério da Saúde. Diretrizes de Atenção à Gestante: a operação Cesariana. 2015. Available from: http://conitec.gov.br/images/Consultas/Relatorios/2015/Relatorio_PCDTCesariana_CP.pdf.

4. Filho BM, Rissin A. A OMS e a epidemia de cesarianas. Rev. Bras. Saude Mater. Infant. 2018; 18 (1): 3-4.

5. Souza CL; Mamédio LJF; Brito MF; Silva VDO; Oliveira KA; Silva ES. Revista Baiana de Saúde Pública 2018; 42 (1): 76-9.

6. Pádua KL, Osis MJD, Faúndes A, Barbosa AH, Moraes OBF. Fatores associados à realização de cesariana em hospitais brasileiros. Rev Saúde Pública 2010; 44(1): 70-9.

7. Granzotto JA, Fonseca SS, Lindemann FL. Fatores relacionados com a mortalidade neonatal em uma Unidade de Terapia Intensiva neonatal na região Sul do Brasil. Rev AMRIGS 2012; 56(1): 57-62.

8. Entringer AP, Pinto M, Dias MAB, Gomes MASM. Análise de custo-efetividade do parto vaginal espontâneo e da cesariana eletiva para gestantes de risco habitual no Sistema Único de Saúde. Caderno de Saúde Pública 2018; 34 (5).

9. Mascarello KC, Horta BL, Silveira MF. Complicações maternas e cesárea sem indicação: revisão sistemática e meta-análise. Rev Saude Publica 2017; 51:105.

10. Osava RH, Silva FMB, Tuesta EF, Oliveira SMJV, Amaral MCE. Caracterização das cesarianas em centro de parto normal. Rev Saúde Pública 2011; 45(6): 1036-43.

11. Agência Nacional de Saúde Suplementar (ANS). Resolução Normativa - RN no 368 , de 6 de janeiro de 2015. Dispõe sobre o direito de acesso à informação das beneficiárias aos percentuais de cirurgias cesáreas e de partos normais, por operadora, por estabelecimento de saúde e por médico e sobre a utilização do partograma, do cartão da gestante e da carta de informação à gestante no âmbito da saúde suplementar. Available from: http://www.ans.gov.br/index2.php?option=com_legislacao\&view=legislacao\&task=TextoLei\&forma $\mathrm{t}=\mathrm{raw} \& \mathrm{id}=2892$.

12. Brasil. Portaria n. 985 de 05 de agosto de 1999. Cria o Centro de Parto Normal, no âmbito do Sistema Único de Saúde, para o atendimento à mulher no período gravídico-puerperal. Diário Oficial da República Federativa do Brasil, Brasília (DF), de 6 agosto de 1999: seção 1: p. 51-2.) [cited 2017 July 20]; Available from:http://www.ans.gov.br/component/legislacao/?view=legislacao\&task=TextoLei\&format=raw\&i $\mathrm{d}=\mathrm{Mjg} 5 \mathrm{Mg}==$.

13. Haddad SEM, Cecatti JG. Estratégias dirigidas aos profissionais para a redução das cesáreas desnecessárias no Brasil. Rev Bras Ginecol Obstet 2011; 33(5):252-62.

14. Barcellos LG, Souza AOR, Machado CAF. Cesariana: uma visão bioética. Revista Bioética 2009; 17(3): 497-510.

15. Novo JLVG, Pellicciari CR, Camargo, LA, Bálsamo SB, Novo NF. Indicações de partos cesáreos em hospitais de atendimento ao Sistema Único de Saúde: baixo e alto riscos. Revista da Faculdade de Ciências Médicas de Sorocaba 2017; 19(2): 67-71.

16. Brasil. Ministério da Saúde. Secretaria de Atenção à Saúde. Departamento de Ações Programáticas Estratégicas. Coordenação Geral de Saúde das Mulheres - DAPES/SAS/MS. Apice On Aprimoramento e Inovação no Cuidado e Ensino em Obstetrícia e Neonatologia. 1. ed. Brasília/DF, agosto de 2017.

17. Fogaça VM, Schneck CA, Riesco MLG. Intervenções obstétricas no trabalho de parto em mulheres submetidas à cesariana. Cogitare Enferm 2007; 12(3): 296-305.

18. Oliveira TA, Aquino MMA, Mariani Neto C. Indução do parto em pacientes com cesárea anterior. 
Femina 2009; 37(8): 427-32.

19. Copen CE, Thoma ME, Kirmeyer S. Interpregnancy Intervals in the United States: Data From the Birth Certificate and the National Survey of Family Growth. National Vital Statistics Reports 2015; 64(3):1-11.

20. Souza ASR, Costa AAR, Coutinho I, Noronha Neto C, Amorim MMR. Indução do trabalho de parto: conceitos e particularidades. Femina 2010; 38(4): 185-94.

21. Souza ASR, Amorim MMR, Porto AMF. Indicações de cesariana baseadas em evidências: parte II. Femina 2010; 38(9): 459-68.

22. Santos LM, Pasquini VZ. A importância do Índice de Apgar. Rev Enferm UNISA 2009; 10(1): 3943.

23. Linhares JJ, Macêdo NM, Arruda GM, Vasconcelos JL, Saraiva TV, Ribeiro AF, Fatores associados à via de parto em mulheres com pré-eclâmpsia. Rev Bras Ginecol Obstet 2014; 36(6): 259-63.

24. Sociedade Brasileira de Pediatria; Sociedade Brasileira de Genética Médica; Federação Brasileira de Associações de Ginecologia e Obstetrícia. Projeto Diretrizes. Recém-nascido macrossômico. Brasília: AMB; CFM [Internet]. 2011 [cited 2017 July 20]; 8 p. Available from: https://diretrizes.amb.org.br/_BibliotecaAntiga/recem nascido_macrossomico.pdf. 